\title{
Yeast capsules for targeted delivery: the future of nanotherapy?
}

\author{
“...targeted delivery via the patient-friendly and cost- \\ effective oral route has been considered to be the holy \\ grail of nanomedicine, due to its multiple benefits, such as, \\ convenience, relatively good safety profile, high patient \\ acceptance and adherence, and easy modulation on dosing."
}

First draft submitted: 15 February 2017; Accepted for publication: 17 February 2017; Published online: 25 April 2017

Keywords: inflammation-associated diseases $\bullet$ macrophage $\bullet$ nanotherapy $\bullet$ oral delivery - yeast capsules

\section{Current challenges for the development of targeted nanotherapies}

Over the past two decades, nanotherapies have been extensively investigated for the treatment of many devastating diseases, including cancers [1], cardiovascular disease [2], diabetes [3] as well as inflammatory and neurodegenerative disorders [4]. The advantages of nanotherapies are largely attributed to their capabilities of overcoming a series of biopharmaceutical, pharmacokinetic and pharmacological limitations related to conventional drug formulations [5-7], thereby leading to improved therapeutic efficacies and reduced side effects. Besides increasing the solubility of lipophilic drugs and enhancing the stability of biological therapeutics, nanotherapies are advantageous at surmounting different physiological and pathological barriers within diseased tissues to achieve site-specific delivery of a diverse array of drugs with distinctive molecular structures and varied sizes $[6,8]$. In addition, nanotherapies can be engineered to selectively release their cargoes in subcellular organelles or under pathological conditions [9], by tailoring the dissolution or hydrolysis performance of nanocarriers via sophisticated chemical approaches. Rapid development in this multidisciplinary field has afforded a myriad of nanotherapies derived from different nanomaterials, such as, liposomes, lipid nanoparticles, polymeric micelles, polymer nanoparticles as well as metallic and inorganic nanostructures. Among them, several anticancer nanomedicines have been approved for clinical use, while many others are in clinical trials [1].

Despite these technological advances, there are numerous challenges in the development of effective, safe and translational nanomedicines for targeted therapy. For the majority of anticancer nanotherapies, in other words, the most broadly and intensively studied nanoformulations, their targeting capacity are mainly based on the enhanced permeability and retention effect of tumor blood vessels [10]. Unfortunately, targeting efficiency of this approach may be considerably compromised by many pathophysiological obstacles, such as, undesirable surface modification by blood components [11], phagocytic clearance [12], poor vasculature and/or limited vascular permeability [13], and high interstitial fluid pressure in solid tumors [14]. Of note, the similar physiological barriers exist for intravenously (iv.) administered nanotherapies in the management of other diseases. These issues may largely contribute to the extremely low delivery efficiency of targeted nanoparticles to malignant tissues [15]. Whereas some challenges can be partly addressed by delicate manipulation of physicochemical characters of nanothera-

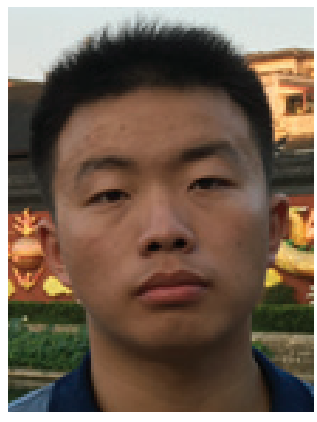

Xiankang $\mathrm{Hu}$

Department of Pharmaceutics, College of Pharmacy, Third Military Medical University, Chongqing 400038, China and

The First Clinical College, Chongqing Medical University, Chongqing 400016, China

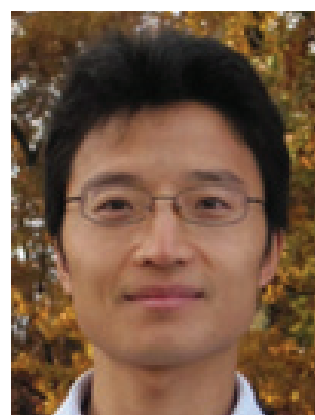

Jianxiang Zhang

Author for correspondence: Department of Pharmaceutics, College of Pharmacy, Third Military Medical University, Chongqing 400038, China jxzhang1980@gmail.com

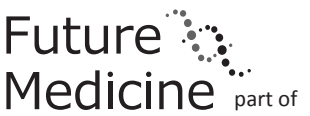


pies, such as, size, shape and surface chemistry, many of them face regulatory hurdles and scrutiny as well as limited cost-effectiveness with respect to clinical translation $[1,16]$. Moreover, iv. injection has been adopted for the majority of currently developed nanomedicines, which need strict regulations due to safety concerns, since delivered nanoparticles may be directly exposed to numerous biomolecular and cellular components in the bloodstream and therefore affect different organs and tissues. Besides, the invasive feature of iv. injection frequently results in poor patient compliance, particularly for long-term treatment of chronic diseases. Consequently, other creative and innovative strategies remain to be discovered.

\section{Yeast microcapsules for targeted oral delivery of nanomedicines}

In contrast to administration of nanotherapies via iv. injection, targeted delivery via the patient-friendly and cost-effective oral route has been considered to be the holy grail of nanomedicine, due to its multiple benefits, such as, convenience, relatively good safety profile, high patient acceptance and adherence, and easy modulation on dosing. Although a large number of nanosystems have been studied for pharmaceutical intervention of different diseases through oral delivery, their capability of targeting distant diseased sites is extremely poor [17]. Accordingly, development of new strategies for enhancing site-specific delivery efficiency after oral administration remains a focus of intense research thus far. Recently, Ostroff and coworkers found that oral delivery of siRNA via yeast shells effectively suppressed systemic inflammation by targeting macrophages [18]. Of note, as a type of eukaryotic single-celled microorganisms, yeasts have been widely utilized for food and beverage production for thousands of years. Nevertheless, it remains unclear whether or not yeast-derived microparticles can effectively load different nanotherapies, properly deliver them to the remote diseased sites and finally exert their pharmacological activities.

We hypothesize that the yeast capsule (YC), which is mainly composed of $\beta$-glucan that can be recognized by dectin-1 on $M$ cells, can precisely and safely transport nanoparticles to remote diseased tissues via macrophage-mediated translocation, thereby negotiating many biological barriers currently preventing effective oral delivery of most nanomedicines invented so far. To provide a conceptual proof study, we developed a new approach to package different nanoparticles into yeast-derived microcapsules most recently [19]. In this strategy, empty YCs were fabricated by removing the major cytoplasmic contents in Saccharomyces cerevisiae via different physicochemi- cal treatments. Thus obtained YCs, with mean size of $4-5 \mu \mathrm{m}$, can directly and efficiently encase positively charged nanoparticles including quantum dots, iron oxide nanoparticles and polymeric nanoparticles. Alternatively, negatively charged nanoparticles may be loaded by pretreatment of YCs with polycations, indicating high diversity of this loading approach. Through this electrostatic force mediated spontaneous deposition, charged nanoparticles with the diameter up to approximately $750 \mathrm{~nm}$ can be effectively loaded into YCs. Moreover, positively charged antiinflammatory or anticancer nanotherapies, which can be easily prepared by a facile one-pot assembly approach, may also be effectively packaged into YCs. After oral administration, nanoparticles packaged in YCs are first transcytosed by M cells and sequentially endocytosed by monocytes/macrophages, subsequently transported to neighboring lymphoid tissues and finally delivered to the distant sites of acute inflammation and tumor in both mouse and rat models. Notably, targeting efficiency of nanoparticles delivered by YCs was comparable or even higher than that of control nanoparticles administered by iv. injection in the examined rodent models of acute inflammation. Through this intrinsic transportation route, nanotherapies enveloped in YCs can be preferentially delivered to diseased sites, affording remarkably improved efficacies for treatment of acute arthritis and tumor, when compared with free nanotherapies. In addition, our preliminary studies demonstrated that nanotherapies orally delivered via YCs can target aortic atherosclerotic plaques, which are generally formed due to chronic inflammation in large- and mediumsized arteries, and are predominantly responsible for fatal cardiovascular and cerebrovascular diseases, such as, myocardial infarction and stroke. Moreover, preliminary acute toxicity evaluation in mice revealed that oral administration of YC at extremely high dose displayed good safety profile, in line with the fact that specific preparations of yeast-derived $\beta$-glucans have been accepted as food ingredients by European Food Safety Authority [20]. Consequently, YCs may serve as functional carriers for targeted oral delivery of a diverse array of nanotherapies in the management of many inflammation-associated diseases.

\section{Future perspective}

Whereas current findings by our group and other researchers revealed YCs possess great potential for oral delivery of nanotherapies targeting distant disease sites, comprehensive studies are necessary from the viewpoint of clinical translation. First, the wall composition of YCs needs to be thoroughly characterized, which is crucial for recognition by $M$ cells and sub- 
sequent endocytosis by monocytes/macrophages and therefore it is intimately related to delivery efficiency. In this aspect, the effects of yeast cell lines and treatment conditions on surface chemistry of YCs must be elucidated. Second, the exact translocation pathways of YCs should be addressed after oral administration in both healthy and diseased animal models. In particular, understanding of targeting mechanisms in different models of inflammation-associated diseases is of great importance for further improving delivery efficiency and avoiding off-target effects. Third, for the development of specific nanotherapies delivered by YCs, 'a $4 \mathrm{R}$ principle' of right therapeutics, right diseases, right combinations and right dosing regimens is suggested to be carefully followed to maximize efficacies and minimize toxicity. Following extensive $R \& D$, we believe that YC-mediated oral delivery of nanomedicines can be advanced into a competitive precision strategy for targeted therapy of many inflammation-associated diseases. Further, the positive finding on YC-mediated

\section{References}

1 Shi J, Kantoff PW, Wooster R et al. Cancer nanomedicine: progress, challenges and opportunities. Nat. Rev. Cancer 17(1), 20-37 (2017).

2 Karimi M, Zare H, Bakhshian Nik A et al. Nanotechnology in diagnosis and treatment of coronary artery disease. Nanomedicine 11(5), 513-530 (2016).

3 Veiseh O, Tang BC, Whitehead KA et al. Managing diabetes with nanomedicine: challenges and opportunities. Nat. Rev. Drug Discov. 14(1), 45-57 (2015).

4 Gendelman HE, Anantharam V, Bronich T et al. Nanoneuromedicines for degenerative, inflammatory, and infectious nervous system diseases. Nanomedicine 11(3), 751-767 (2015).

5 Zuckerman JE, Davis ME. Clinical experiences with systemically administered siRNA-based therapeutics in cancer. Nat. Rev. Drug Discov. 14(12), 843-856 (2015).

6 Blanco E, Shen H, Ferrari M. Principles of nanoparticle design for overcoming biological barriers to drug delivery. Nat. Biotechnol. 33(9), 941-951 (2015).

7 Cabral H, Kataoka K. Progress of drug-loaded polymeric micelles into clinical studies. J. Control. Release 190, 465-476 (2014).

8 von Roemeling C, Jiang W, Chan CK et al. Breaking down the barriers to precision cancer nanomedicine. Trends Biotechnol. 35(2), 159-171 (2017).

9 Lu Y, Aimetti AA, Langer R et al. Bioresponsive materials. Nat. Rev. Mater. 2, 16075 (2016).

10 Fang J, Nakamura H, Maeda H. The EPR effect: unique features of tumor blood vessels for drug delivery, factors involved, and limitations and augmentation of the effect. Adv. Drug Deliv. Rev. 63(3), 136-151 (2011). oral delivery inspired us that development of targeted nanotherapies by integrating currently available nanomaterials and biochemically engineered microorganisms, in combination with cellular and biomolecular components of the innate and adaptive immune systems, is an intriguing approach, by taking advantage of their specific translocation mechanisms or processes and using them in the right way.

\section{Financial \& competing interests disclosure}

This work was financially supported by the National Natural Science Foundation of China (no. 81471774) and the Program for New Century Excellent Talents in University (no. NCET13-0703). The authors have no other relevant affiliations or financial involvement with any organization or entity with a financial interest in or financial conflict with the subject matter or materials discussed in the manuscript apart from those disclosed.

No writing assistance was utilized in the production of this manuscript.

11 Salvati A, Pitek AS, Monopoli MP et al. Transferrinfunctionalized nanoparticles lose their targeting capabilities when a biomolecule corona adsorbs on the surface. Nat. Nanotechnol. 8(2), 137-143 (2013).

12 Rodriguez PL, Harada T, Christian DA et al. Minimal "self" peptides that inhibit phagocytic clearance and enhance delivery of nanoparticles. Science 339 (6122), 971-975 (2013).

13 Chauhan VP, Stylianopoulos T, Martin JD et al. Normalization of tumour blood vessels improves the delivery of nanomedicines in a size-dependent manner. Nat. Nanotechnol. 7(6), 383-388 (2012).

14 Swartz MA, Lund AW. Lymphatic and interstitial flow in the tumour microenvironment: linking mechanobiology with immunity. Nat. Rev. Cancer 12(3), 210-219 (2012).

15 Wilhelm S, Tavares AJ, Dai Q et al. Analysis of nanoparticle delivery to tumours. Nat. Rev. Mater. 1, 16014 (2016).

16 Venditto VJ, Szoka FC Jr. Cancer nanomedicines: so many papers and so few drugs! Adv. Drug Deliv. Rev. 65(1), 80-88 (2013).

17 Mitragotri S, Burke PA, Langer R. Overcoming the challenges in administering biopharmaceuticals: formulation and delivery strategies. Nat. Rev. Drug Discov. 13(9), 655-672 (2014).

18 Aouadi M, Tesz GJ, Nicoloro SM et al. Orally delivered siRNA targeting macrophage Map4k4 suppresses systemic inflammation. Nature 458(7242), 1180-1184 (2009).

19 Zhou X, Zhang XJ, Han SL et al. Yeast microcapsulemediated targeted delivery of diverse nanoparticles for imaging and therapy via the oral route. Nano Lett. 17(2), 1056-1064 (2017).

20 Samuelsen AB, Schrezenmeir J, Knutsen SH. Effects of orally administered yeast-derived beta-glucans: a review. Mol. Nutr. Food Res. 58(1), 183-193 (2014). 